\title{
Patient Portal Use and Blood Pressure Control in Newly Diagnosed Hypertension
}

\author{
William Manard, MD, Jeffrey F. Scherrer, PhD, Joanne Salas, MPH, and \\ F. David Schneider, MD
}

Background: Current evidence that patient portal use improves disease management is inconclusive. Randomized controlled trials have found no benefit of Web-based patient-provider communication for blood pressure (BP) control, but patients from these studies were not selected for uncontrolled hypertension, nor did measures of portal use occur in a real-world setting, as captured in the electronic medical record. This study determined whether patient portal use by patients with treated, incident hypertension was associated with achieving BP control.

Methods: Between 2008 to 2010, 1571 patients with an incident hypertension diagnosis, ages 21 to $>89$ years, were identified from an academic medical center primary care patient data registry. Cox proportional hazard models were computed to estimate the association between portal use and incident BP control during follow-up (2011-2015), before and after adjusting for covariates. Covariates included sociodemographics, smoking, obesity and other physical and mental health comorbidities, and volume of health care utilization.

Results: After adjusting for age, portal users were more likely than nonusers to achieve BP control (hazard ratio, 1.24; 95\% confidence interval, 1.06-1.45). After adjustment for sociodemographics, portal use was no longer associated with BP control (hazard ratio, 0.98 ; 95\% confidence interval, $0.83-$ 1.16).

Conclusions: Patient sociodemographic factors, including race, sex, and socioeconomic status, account for the observation that portal use leads to BP control among persons with newly diagnosed hypertension. Further research is warranted to determine whether there are benefits of portal use for other chronic conditions.(J Am Board Fam Med 2016;29:452-459.)

Keywords: Academic Medical Centers, Blood Pressure, Blood Pressure Determination, Chronic Disease, Comorbidity, Disease Management, Electronic Health Records, Follow-Up Studies, Personal Health Records, Hypertension, Internet, Mental Health, Obesity, Patient Care, Primary Health Care, Proportional Hazards Models, Randomized Controlled Trials as Topic, Registries, Smoking, Social Class

Approximately 70 million people in the United States have hypertension, and this number is expected to increase as the population ages. ${ }^{1}$ Furthermore, the rate of blood pressure (BP) control is

This article was externally peer reviewed.

Submitted 6 January 2016; revised 3 March 2016; accepted 8 March 2016.

From the Department of Family and Community Medicine, Saint Louis University School of Medicine, St. Louis, MO.

Funding: A Mindlin Foundation Education grant provided partial support (to JFS).

Conflict of interest: none declared.

Corresponding author: William Manard, MD, Department of Family and Community Medicine, Saint Louis University School of Medicine, 1402 South Grand Blvd, St. Louis, MO 63104 (E-mail: manardwt@slu.edu). below the HealthyPeople 2010 goal (NHLBI). ${ }^{2}$ As a major risk factor for cardiovascular disease, this trend is problematic from a public health perspective.

Antihypertensive medications and lifestyle modification require consistency to be effective. Follow-up appointments with a physician or other health care providers should happen at regular intervals to monitor progress and adjust medication dosages accordingly until the $\mathrm{BP}$ goal is reached. ${ }^{3}$ After the patient's BP is controlled, follow-up visits may be spaced farther apart. ${ }^{3}$ Therefore, ongoing patient communication with their providers can aid patients in treating hypertension by allowing for medication adjustments as necessary and patient 
questions on lifestyle modifications to be addressed between appointments.

Patient portals within electronic health records provide a unique opportunity for patients to be involved in their care and to communicate with their provider between appointments. ${ }^{4} \mathrm{~A}$ recent systematic review of the impact of patient portals on chronic disease management concluded that the evidence is insufficient to draw conclusions regarding their benefit. ${ }^{5}$ At this time, we are unaware of any studies that have investigated patient portal use on outcomes among patients with a new diagnosis of hypertension. The few reports comparing the use and nonuse of patient portals and similar webenhanced communication related to BP control have been in patients with diabetes, ${ }^{6-8}$ and all consistently found no effect of portal use on BP control.

Additional research on $\mathrm{BP}$ outcomes and patient portal use is needed because the literature is inconsistent on the benefits of patient portal use and has been dominated by controlled studies that assigned patients to portal access. ${ }^{9}$ Most of all, in our review of the available literature, there are no reports on outcomes among patients with newly diagnosed hypertension. Therefore, we sought to determine whether patients who voluntarily used a patient portal, compared with those who did not, had a greater likelihood of achieving controlled BP in an academic primary care setting.

\section{Methods \\ Subjects}

The Department of Family and Community Medicine's Primary Care Patient Data Registry (PCPD) at Saint Louis University School of Family Medicine was the source of data. The PCPD consists of all family medicine (FM) and general internal medicine (GIM) patients $(\mathrm{n}=33,661)$ who had at least 1 face-to-face encounter (eg, office visit, procedure visit, or clinical support) between July 1, 2008, and June 30, 2015. Study variables were created from International Classification of Diseases, 9th Revision, Clinical Modification (ICD-9-CM) codes, prescription orders, Current Procedure Terminology 4 codes, social history, family history, demographics, laboratory orders, referrals, and vital signs. The PCPD has been previously described, and additional details on the method of creating the resource are available. ${ }^{10-13}$ The institutional review board approved the creation of this retrospective cohort and its use for primary care research.

The analytic cohort for this analysis was developed by first selecting adult patients aged $\geq 18$ years with a diagnosis of new-onset hypertension between 2008 and 2010 ( $\mathrm{n}=5153)$. The sample was then limited to patients that had uncontrolled hypertension at their last visit before 2011 ( $\mathrm{n}=$ 2073), which was the year the patient portal was implemented. Eligible patients must have had at least 1 visit during follow-up (January 1, 2011, through June 30,$2015 ; \mathrm{n}=1645)$ and have received treatment for hypertension $(n=1600)$. After removing 8 patients with missing race, 2 with missing socioeconomic status (SES), and 20 with missing marital status, the final analytic sample was 1571 patients aged $\geq 23$ years.

\section{Measures}

The exposure variable was patient portal use. In 2010, primary care patients were invited to register for the online portal. This allowed patient access to lab results, vital signs, diagnosis, medication fill requests, and a message interface to communicate with their providers via the Internet. Patients or providers could initiate messages. Patients with orders to monitor blood pressure at home were asked to use an arm BP cuff 2 to 3 times a week, with measures taken 5 minutes apart. Radial and digital artery measures were not allowed. Patients were able to submit discrete $\mathrm{BP}$ values to their provider, but this occurred infrequently, and such data were not stored in the patient's vital sign record, that is, the source of data for this study. For this analysis we created a binary variable indicating active patient portal use (the patient wrote messages) versus no active use (the patient did not register or registered but did not write messages [ie, only read physician messages]).

\section{Outcome: BP Control}

BP measures were obtained from vital sign data available from the electronic medical record. $\mathrm{Pa}$ tients were considered to have uncontrolled hypertension if they met 1 of the following 8th Joint National Committee 2014 guidelines for elevated BP: $150 / 90 \mathrm{mmHg}$ for patients $\geq 60$ years old without diabetes and without chronic kidney disease or $140 / 90 \mathrm{mmHg}$ for all other patients. ${ }^{3}$

Following the 8th Joint National Committee 2014 guidelines, we defined incident control in the 
follow-up period (2011 to 2015) as (1) systolic blood pressure $<150$ and diastolic $<90$ for patients $\geq 60$ years of age without diabetes and without chronic kidney disease, or (2) systolic $<140$ and diastolic $<90$ for all other patients.

\section{Covariates}

Covariates were selected to control for both the voluntary nature of portal use with the assumption that patients who had more comorbidity would be more likely to use the portal. We controlled for smoking, depression, obesity, comorbidity, and volume of health care use, which all impact contact with the health care system and odds of hypertension control. In addition, controlling for FM versus GIM clinic and patient sex, age, race, and SES adjustment improved generalizability and controlled for differences in access to Internet resources; for example, older patients were less likely to have easy Internet access.

Sociodemographic variables available in the medical record included age, race, sex, and marital status. Following a method described by Roblin, ${ }^{14}$ we used ZIP codes to compute SES status. Specifically, the SES index was based on information from 7 measures of SES obtained from 5-year census estimates (2009 to 2013) from the American Community Survey. The 7 variables included (1) the percentage of households with income below the poverty level, (2) the percentage of households receiving public assistance, (3) the percentage of households with an annual income $<\$ 35,000$, (4) the percentage of adult males aged 20 to 64 years not in the labor force, (5) the percentage of adults $\geq 25$ years old with less than a high school education, (6) $\log$ of median household income, and (7) $\log$ of median value of single family homes. Median household income and value of single family homes were $\log$ transformed so that values were on a similar scale to the other 5 measures. Using a principal components analysis of all 33,120 US ZIP codes and their corresponding 7 measures of SES, a standardized factor score was assigned to each US ZIP code. A higher standardized factor score indicates lower SES. Standardized factor scores computed from all US ZIP codes were then matched to ZIP codes of the entire primary care patient population from 2008 to 2015 ( $\mathrm{n}=33,661$ patients; $\mathrm{n}=1326$ ZIP codes). Based on SES factor score distribution in the entire primary care population, eligible patients were assigned into quartiles (eg, lowest, low-middle, upper-middle, and highest SES).

We adjusted for whether patients were seen by providers in FM or GIM. To adjust for the volume of health care use, the average number of clinic visits per month was calculated and the distribution divided into quartiles. Health care use was modeled as a binary variable, with high use representing the top quartile of health care use versus all other levels of use. A history of smoking from social history information or an ICD-9-CM code for nicotine dependence was used to create a never, former, and current smoker (2008 to 2015) variable. Obesity was determined from body mass index or an ICD9-CM code for obesity. The comorbidity index was based on the Charlson Comorbidity Index adapted by Romano et al. ${ }^{15}$ The index is derived from the presence of 17 health conditions associated with morbidity and mortality, with higher scores indicating worse health. ${ }^{15}$ Depression was defined by 2 outpatient visits with an ICD-9-CM code for depression (codes 296.2x, 296.3x, or 311) in the same 12-month period. ${ }^{16,17}$ A single occurrence of an ICD-9-CM code was used to define conditions in the comorbidity index.

\section{Analytic Approach}

Descriptive statistics were computed for bivariate associations between covariates and both portal use and incident BP control. $\chi^{2}$ Tests and independent samples $t$ tests were used to assess the distributions of covariates among portal use and BP control. Hazard ratios for incident BP control were estimated using Cox proportional hazards models. $\mathrm{Pa}-$ tient portal use, obesity, comorbidity index, and depression were treated as time-dependent covariates. Follow-up time was in months. The relationship of age and BP control followed a polynomial relationship, so age-adjusted models included an age + age squared + age cubed term.

Follow-up continued until the first controlled $\mathrm{BP}$ or until the last date of available data (eg, date of the last clinical encounter). The Proc PHREG procedure in SAS version 9.4 (SAS Institute, Cary, NC), with $\alpha$ set at 0.05 , was used for the Cox regression models. Two-tailed tests were conducted to allow for both risk factors and protective effects. To assess the contribution of each individual covariate to the association between portal use and BP control, separate proportional hazard models were assessed, beginning with an age-adjusted 
Table 1. Distribution of Sociodemographics, Covariates, and Incident Blood Pressure Control Among Adult Primary Care Patients with Treated Hypertension and Uncontrolled Blood Pressure at Baseline (2008-2010), by Patient Portal Use During the Follow-up Period (2011-2015) (N = 1571)

\begin{tabular}{|c|c|c|c|c|}
\hline Variable & $\begin{array}{l}\text { Total Cohort } \\
(\mathrm{N}=1571)\end{array}$ & $\begin{array}{l}\text { Patient Portal Nonusers } \\
\qquad(\mathrm{n}=1171)\end{array}$ & $\begin{array}{l}\text { Patient Portal Users } \\
(\mathrm{n}=400)\end{array}$ & $P$ Value \\
\hline Blood pressure control (yes) & $1425(90.7)$ & $1045(89.2)$ & $380(95.0)$ & .001 \\
\hline Age (years), mean (SD) & $64.1(13.3)$ & $65.1(13.5)$ & $60.9(12.2)$ & $<.0001$ \\
\hline Nonwhite race & $931(59.3)$ & $819(69.9)$ & $112(28.0)$ & $<.0001$ \\
\hline Female sex & $988(62.9)$ & $726(62.0)$ & $262(65.5)$ & .211 \\
\hline Married & $632(40.2)$ & $401(34.2)$ & $231(57.7)$ & $<.0001$ \\
\hline SES index (by zip code) & & & & $<.0001$ \\
\hline Lowest & $603(38.4)$ & $530(45.3)$ & $73(18.2)$ & \\
\hline Lower-middle & $347(22.1)$ & $271(23.1)$ & $76(19.0)$ & \\
\hline Upper-middle & $376(23.9)$ & $233(19.9)$ & $143(35.8)$ & \\
\hline Highest & $245(15.6)$ & $137(11.7)$ & $108(27.0)$ & \\
\hline Clinic type $^{1}$ & & & & $<.0001$ \\
\hline GIM & $1224(77.9)$ & $965(82.4)$ & $259(64.8)$ & \\
\hline Family medicine & $347(22.1)$ & $206(17.6)$ & $141(35.2)$ & \\
\hline High clinic utilization & $876(55.8)$ & $632(54.0)$ & $244(61.0)$ & .015 \\
\hline Smoking status, 2008-2015 & & & & $<.0001$ \\
\hline Never & $758(48.3)$ & $537(45.9)$ & $221(55.2)$ & \\
\hline Former & $403(25.6)$ & $295(25.2)$ & $108(27.0)$ & \\
\hline Current & $410(26.1)$ & $339(28.9)$ & $71(17.8)$ & \\
\hline Depression & $262(16.7)$ & $179(15.3)$ & $83(20.8)$ & .011 \\
\hline Obesity & $1051(66.9)$ & $772(65.9)$ & $279(69.7)$ & .161 \\
\hline $\begin{array}{l}\text { Comorbidity index, mean } \\
\text { (SD) }\end{array}$ & $2.1(2.4)$ & $2.3(2.4)$ & $1.6(2.3)$ & $<.0001$ \\
\hline
\end{tabular}

Data are $\mathrm{n}(\%)$ unless otherwise indicated.

GIM, general internal medicine; SD, standard deviation; SES, socioeconomic status.

base model assessing the bivariate relationship between portal use and BP control. Separate models added each individual covariate to the base model to assess the impact on the hazard ratio for the relationship of portal and BP control; last, a fully adjusted model containing all covariates was computed.

\section{Results}

On average, patients were 64.1 years old (standard deviation, 13.3 years); the majority were women $(62.9 \%)$ and nonwhite race $(59.3 \%)$, and $40.2 \%$ were married. As shown in Table 1, among this patient cohort, $74.5 \%(\mathrm{n}=1171)$ actively used the patient portal, and the remainder either did not register or registered but did not actively use it. During follow-up, $90.7 \%$ of the total cohort achieved BP control. Significantly more patients achieved BP control if they used the portal compared with those who did not $(95.0 \%$ vs $89.2 \% ; P=.001)$. Compared with nonusers, portal users were significantly younger $(P<.0001)$, more often white $(P<.0001)$, and more often married $(P<.0001)$. Portal users versus nonusers were significantly from uppermiddle to highest SES $(P<.0001)$. Portal use was more common among FM patients than GIM patients $(P<.0001)$, and users were more often high health care utilizers $(P=.015)$. Portal use was less common among current smokers $(P<.0001)$. Portal users were more likely to have depression $(P=.011)$ and have significantly lower comorbidity scores $(P<.0001)$.

The distribution of covariates by BP control during follow-up are shown in Table 2. Patients who achieved control, compared with those remaining uncontrolled, were significantly older $(P<$ $.0001)$, less often of minority race $(P<.001)$, and more likely to be married $(P=.038)$. BP control was positively associated with higher SES $(P=$ $.003)$ and with high health care utilization $(P<$ .0001). Patients who achieved control were signif- 
Table 2. Distribution of Sociodemographics and Covariates Among Adult Primary Care Patients with Treated Hypertension and Uncontrolled Blood Pressure at Baseline (2008-2010), by Patient Portal Use During the FollowUp Period (2011-2015) ( $=1571)$

\begin{tabular}{|c|c|c|c|}
\hline Variable & $\begin{array}{c}\text { No BP } \\
\text { Control } \\
(\mathrm{n}=146)\end{array}$ & $\begin{array}{l}\text { BP Control } \\
(\mathrm{n}=1425)\end{array}$ & $P$ Value \\
\hline Age (years), mean (SD) & $59.8(14.3)$ & $64.5(13.1)$ & $<.0001$ \\
\hline Nonwhite race & $105(71.9)$ & $826(58.0)$ & .001 \\
\hline Female sex & $86(58.9)$ & $902(63.3)$ & .295 \\
\hline Married & $47(32.2)$ & $585(41.1)$ & .038 \\
\hline SES index (by zip code) & & & .003 \\
\hline Lowest & $76(52.0)$ & $527(37.0)$ & \\
\hline Lower-middle & $30(20.6)$ & $317(22.3)$ & \\
\hline Upper-middle & $25(17.1)$ & $351(24.6)$ & \\
\hline Highest & $15(10.3)$ & $230(16.1)$ & \\
\hline Clinic type $^{1}$ & & & .794 \\
\hline GIM & $115(78.8)$ & $1109(77.8)$ & \\
\hline Family Medicine & $31(21.2)$ & $316(22.2)$ & \\
\hline High clinic utilization & $24(16.4)$ & $852(59.8)$ & $<.0001$ \\
\hline Smoking status, 2008-2015 & & & .023 \\
\hline Never & $82(56.2)$ & $676(47.4)$ & \\
\hline Former & $24(16.4)$ & $379(26.6)$ & \\
\hline Current & $40(27.4)$ & $370(26.0)$ & \\
\hline Depression & $10(6.8)$ & $252(17.7)$ & .001 \\
\hline Obesity & $92(63.0)$ & $959(67.3)$ & .295 \\
\hline Comorbidity index, mean (SD) & $1.7(2.0)$ & $2.2(2.5)$ & .007 \\
\hline
\end{tabular}

Data are $\mathrm{n}(\%)$ unless otherwise indicated.

$\mathrm{BP}$, blood pressure; GIM, general internal medicine; SD, standard deviation; SES, socioeconomic status.

icantly more likely to be former smokers $(P=$ $.023)$, have a diagnosis for depression $(P<.001)$, and have a significantly higher comorbidity index score $(P=.007)$.

Results of age-adjusted, bivariate regression models estimating the association between portal use, covariates, and incident BP control during follow-up are shown in Table 3. Patients who used the portal, compared with nonusers, were $24 \%$ more likely to achieve BP control (hazard ratio [HR], 1.24 ; $95 \%$ confidence interval $[\mathrm{CI}], 1.06-1.45)$. Patients of a minority race were significantly less likely to achieve BP control (HR, 0.79; 95\% CI, 0.71-0.88). Female patients (HR, 1.13; 95\% CI, 1.02-1.26) and those who were married (HR, 1.12; 95\% CI, 1.011.24) were significantly more likely to have BP control. Patients in the upper-middle and highest SES categories were about $30 \%$ more likely to achieve BP control compared with those with the lowest SES. FM patients were more likely to achieve BP control (HR, 1.12; 95\% CI, 1.02-1.31), and high health care utilizers had greater risk of BP control (HR, 1.76;
95\% CI, 1.58-1.96). Last, patients with depression were significantly more likely to achieve BP control (HR, 1.35; 95\% CI, 1.16-1.58).

Results of Cox proportional hazard models are shown in Table 4 . In the base model adjusting only for age, patients who were active portal users were $24 \%$ more likely to gain BP control compared with nonactive users. Five separate models adjusting for race, sex, SES index, clinic type, and volume of health care utilization all resulted in a smaller and nonsignificant hazard ratio (HR range, 1.11-1.16). Models that separately adjusted for depression, obesity, and comorbidity index and a multivariate with all these health conditions included resulted in HRs similar to the base model (HR range, 1.22-1.24). Multivariate models that simultaneously adjusted for demographics, clinic setting, and utilization and the full model both resulted in a nonsignificant association between active portal use and BP control (HR, 0.98; 95\% CI, 0.83-1.16 and HR, 0.97; 95\% CI, 0.82-1.15, respectively). 
Table 3. Age-Adjusted Bivariate Associations of Patient Portal Use, Sociodemographics, and Covariates with Incident Blood Pressure Control Among Adult Primary Care Patients with Treated Hypertension and Uncontrolled Blood Pressure at Baseline $(\mathrm{N}=1571)$

\begin{tabular}{lc}
\hline Variable & Bivariate HR (95\% CI) \\
\hline Portal activity (yes) & $1.24(1.06-1.45)$ \\
Age (years) & $0.92(0.80-1.06)$ \\
Nonwhite race & $0.79(0.71-0.88)$ \\
Female sex & $1.13(1.02-1.26)$ \\
Married & $1.12(1.01-1.24)$ \\
SES index (by zip code) & \\
Lowest & 1.00 \\
Lower-middle & $1.11(0.97-1.28)$ \\
$\quad$ Upper-middle & $1.30(1.14-1.49)$ \\
$\quad$ Highest & $1.33(1.14-1.55)$ \\
Clinic type & \\
$\quad$ GIM & 1.00 \\
$\quad$ Family medicine & $1.16(1.02-1.31)$ \\
High clinic utilization & $1.76(1.58-1.96)$ \\
Smoking status, 2008-2015 & \\
$\quad$ Never & 1.00 \\
Former & $1.12(0.98-1.27)$ \\
Current & $1.09(0.96-1.24)$ \\
Depression & $1.35(1.16-1.58)$ \\
Obesity & $1.07(0.96-1.19)$ \\
Comorbidity index & $1.01(0.98-1.04)$ \\
\hline
\end{tabular}

CI, confidence interval; GIM, general internal medicine; HR, hazard ratio; SES, socioeconomic status.

\section{Discussion}

In a retrospective cohort of 1571 patients with uncontrolled hypertension between 2008 and 2010, active patient portal users were $24 \%$ more likely to achieve $\mathrm{BP}$ control than nonusers during follow-up in 2011 to 2015. However, after adjusting for sociodemographic factors, this association was no longer present (HR, 0.98; 95\% CI, 0.83-1.16). However, adjusting for health conditions and comorbidity index did not contribute to the association between portal use and BP control. To our knowledge, these results are novel in determining whether patient portal use is associated with $\mathrm{BP}$ control in patients with newly diagnosed hypertension. Overall, the relationship between portal use and BP control is explained by demographic factors, whether patients are FM or GIM users, and total health care utilization. Our results are consistent with previous findings that portal use, and other electronic communication, was not associated with $\mathrm{BP}$ control among patients with diabetes. ${ }^{6-8} \mathrm{~A}$
Table 4. Association Between Patient Portal Use and Incident Blood Pressure Control Adjusting for Each Covariate in a Cohort of Adult Primary Care Patients with Treated Hypertension and Uncontrolled Blood Pressure at Baseline $(\mathrm{N}=1571)$

\begin{tabular}{lc}
\hline Model $^{*}$ & Portal Activity, HR (95\% CI ${ }^{\dagger}$ \\
\hline Base model (portal activity) & $1.24(1.06-1.45)$ \\
Base + race & $1.13(0.96-1.33)$ \\
Base + sex & $1.14(1.02-1.27)$ \\
Base + marital status & $1.22(1.04-1.43)$ \\
Base + SES index & $1.16(0.99-1.36)$ \\
Base + clinic type & $\neq$ \\
Base + utilization & $1.13(0.99-1.28)$ \\
Base + smoking & $1.11(0.95-1.30)$ \\
Base + depression & $1.24(1.05-1.45)$ \\
Base + obesity & $1.23(1.05-1.44)$ \\
Base + comorbidity index & $1.24(1.06-1.45)$ \\
Base + race + gender + & $1.24(1.06-1.46)$ \\
$\quad$ marital status + SES & $0.98(0.83-1.16)$ \\
index + clinic type + & \\
utilization & \\
Base + smoking + & \\
$\quad$ depression + obesity + & \\
comorbidity index & \\
Base + all covariates & \\
\hline
\end{tabular}

*All models are adjusted for age: age + age (squared) + age (cubed).

${ }^{\dagger}$ All hazard ratios (HRs) and confidence intervals (CIs) were calculated using Cox proportional hazards models.

${ }^{\ddagger}$ Clinic types included general internal medicine and family medicine.

SES, socioeconomic status.

systematic review of patient portals and outcomes found weak evidence that portal use is associated with better patient outcomes, including improved self-care, medication adherence, and chronic disease management. ${ }^{18}$ Using data from the same source as used in this study, we recently reported a positive association between greater volume of portal use and better glycemic control. ${ }^{19}$ However, this cross-sectional study did not allow us to determine whether the association was due to portal use leading to better diabetes management, or whether patients with better diabetes management were more likely to use the patient portal. Given the variety of methodologies and study designs and varying definitions of portal use in the extant literature, additional research designed to detect chronic disease outcomes using a standard definition of portal use is needed.

Because patient portal use alone is associated with BP control, factors associated with not using 
the portal may explain adjusted results. Only 28\% of nonwhites used the portal, and only $18.2 \%$ of the patients from the lowest SES and 19.0\% from the lower-middle SES used the portal, compared with $35.8 \%$ and $27.0 \%$ from the 2 highest SES categories. We speculate that low rates of portal use among minorities and disadvantaged patients contribute to a decreased likelihood of achieving BP control.

The lower portal use in GIM compared with FM may be because of the presence of the medical home in FM, which encourages more communication and preventive care. Patients who are high utilizers are both more likely to achieve BP control and use the patient portal, suggesting that detection bias accounts for much of the portal use-BP control association. Despite this limitation, our results point to a health disparity related to SES and race. Targeted interventions to disadvantaged minority patients may include education about portal communication, but this will only be effective if access to the Internet is equitable.

These results are limited in several ways. First, the patient population is from a Midwestern academic medical center and may not generalize to other parts of the country. Data on what components of the portal were used by patients are not available, and we are unable to determine whether users utilized all available information (eg, medications prescribed, laboratory results, vital signs). It is possible that among users, those who take advantage of all aspects of the patient portal would still benefit in terms of BP control. Change in home access to the Internet has increased rapidly from 2009 , when $68.7 \%$ had home Internet, to 2013 , when $83.8 \%$ of households had Internet. ${ }^{20,21} \mathrm{We}$ did not account for potential yearly variation in changing access to patient portals, and it is unknown whether increasing availability, especially for high-speed Internet, will result in a greater contribution of patient portals to disease management. BP control could be misclassified if patients had good control at home but not when BP is measured in the physician's examination room. Last, medical record data do not contain information on beliefs and attitudes about medical care, self-care, and using electronic sources to communicate with physicians. Thus, these results may change if adjusted for individual preferences that may influence portal use and self-care. To partially address this limitation, we conducted further eval- uation of portal access, comparing average BP control for the subset of active and nonactive subjects in the sample who used the primary care clinics before implementation of the patient portal in 2008 and $2009(\mathrm{n}=1282)$. The prevalence of BP control among those who eventually activated $(n=423)$ their portal access was $54.6 \%$ versus $43.7 \%$ among those who remained inactive $(\mathrm{n}=859 ; P<.001)$. The mean systolic pressure for activators was 138.3 , versus 141.4 among nonactivators $(P<$ .001 ), and mean diastolic was 82.3 versus 81.8 for activators and nonactivators, respectively $(P=.41)$. These results suggest our findings may be partly explained by a slightly healthier subsample among eventual portal users compared with persistent nonusers. However, given the minimal size of mean difference in systolic and diastolic pressures in 2008 to 2009 , it would seem unlikely that incident BP control could be entirely explained by patient BP characteristics before portal availability.

\section{Conclusions}

Our results are inconclusive, as is the current literature, ${ }^{5}$ and prevent the conclusion that patient portal use alone accounts for BP control. However, we uncovered some evidence that a health disparity exists in the use of patient portals and its benefits for BP control. Additional research is needed to determine which sociodemographic groups benefit the most from access to patient portals and what conditions and what outcomes are most sensitive to improvement via portal use.

\section{References}

1. Centers for Disease Control and Prevention. High blood pressure fact sheet. Available from: http:// www.cdc.gov/dhdsp/data_statistics/fact_sheets/docs/ fs_bloodpressure.pdf. Accessed May 28, 2015.

2. Centers for Disease Control and Prevention. Healthy People 2010: objectives for improving health. Section 12: heart disease and stroke. Available from: http://www.cdc.gov/dhdsp/docs/hp2010.pdf. Accessed May 28, 2015.

3. James PA, Oparil S, Carter BL, et al. Evidence-based guideline for the management of high blood pressure in adults: report from the panel members appointed to the Eighth Joint National Committee (JNC 8). JAMA 2014;311:507-20.

4. Wade-Vuturo A, Mayberry L, Osborn C. Secure messaging and diabetes management: experiences and perspectives of patient portal users. J Am Med Inform Assoc 2013;20:519-25. 
5. Goldzweig CL, Orshansky G, Shekelle P, et al. Electronic patient portals: evidence on health outcomes, satisfaction, efficiency, and attitudes. Ann Intern Med 2013;159:677-700.

6. Tang PC, Overhage J, Young C, et al. Online disease management of diabetes: Engaging and Motivating Patients Online With Enhanced Resources-Diabetes (EMPOWER-D), a randomized controlled trial. J Am Med Inform Assoc 2013;20:526-34.

7. Ralston JD, Hirsch I, Hoath J, et al. Web-based collaborative care for type 2 diabetes: a pilot randomized trial. Diabetes Care 2009;32:234-9.

8. Grant RW, Wald JS, Schnipper JL, et al. Practicelinked online personal health records for type $2 \mathrm{di}$ abetes mellitus: a randomized controlled trial. Arch Intern Med 2008;168:1776-82.

9. Ammenwerth E, Schnell-Inderst P, Hoerbst A. The impact of electronic patient portals on patient care: a systematic review of controlled trials. J Med Internet Res 2012;14:e162.

10. Scherrer JF, Salas J, Brieler JA, Miller BJ, Meyer D, Schneider FD. Depression leads to incident vascular disease: evidence for the relevance to primary care. Fam Pract 2015;32:147-51.

11. Hooks-Anderson DR, Crannage EF, Salas J, Scherrer JF. Race and referral to diabetes education in primary care patients with prediabetes and diabetes. Diabetes Educ 2015;41:281-9.

12. Brieler JA, Scherrer JF, Salas J. Differences in prescribing patterns for anxiety and depression between general internal medicine and family medicine. J Affect Disord 2015;172:153-8.

13. Schoen MW, Salas J, Scherrer JF, Buckhold FR. Cholesterol treatment and changes in guidelines in an academic medical practice. Am J Med 2015;128: 403-9.

14. Roblin DW. Validation of a neighborhood SES index in a managed care organization. Med Care 2013; 51:1-8.

15. Romano PS, Roos LL, Jollis JG. Adapting a clinical comorbidity index for use with ICD-9-CM administrative data: differing perspectives. J Clin Epidemiol 1993;46:1075-9.

16. Frayne SM, Miller DR, Sharkansky EJ, et al. Using administrative data to identify mental illness: what approach is best? Am J Med Qual 2010;25:42-50.

17. Solberg LI, Engebretson KI, Sperl-Hillen JM, Hroscikoski MC, O'Connor PJ. Are claims data accurate enough to identify patients for performance measures or quality improvement? The case of diabetes, heart disease, and depression. Am J Med Qual 2006;21:238-45.

18. Kruse CS, Bolton K, Freriks G. The effect of patient portals on quality outcomes and its implications to meaningful use: a systematic review. J Med Internet Res 2015;17:e44.

19. Devkota B, Salas J, Sayavong S, Scherrer JF. Use of an online patient portal and glucose control in primary care patients with diabetes. Popul Health Manag 2016;19:125-31.

20. U.S. Census Bureau. Current population survey, October 1984, 1989, 1993, 1997, 2000, 2001, 2003, 2007, 2009. Available from: http://www.census.gov/ hhes/computer/. Accessed December 2015.

21. File T, Ryan C. Computer and internet use in the United States: 2013, American Community Survey Reports, ACS-28. Washington, DC: U.S. Census Bureau; 2014. 